\title{
Effect of benzalkonium chloride on the stability of the precorneal tear film in rabbit and man
}

\author{
WILLIAM S. WILSON, * ALLAN J. DUNCAN, * AND JEFFREY L. JAY† \\ From the Departments of Pharmacology* and Ophthalmology, $\dagger$ Glasgow University, Glasgow
}

The tear film is important both as a lubricating and protective layer and because it provides the outer, optically-smooth, refracting surface of the cornea. The tear film has a thickness of about $6 \mu \mathrm{m}$ and appears to be able to maintain its integrity during long periods between the respreading and replenishing action of the blink, by two means:

I. The corneal epithelium holds the tear film on its surface, probably aided by mucus from conjunctival cells, which encourages spreading of the film (Lemp, Holly, Iwata, and Dohlman, 1970);

2. The watery component of the tear film is covered by an outer layer of oil, secreted by the Meibomian glands, which reduces loss by evaporation (Mishima and Maurice, 196r; Iwata, Lemp, Holly, and Dohlman, 1969).

Little attention has hitherto been given to the possibility that topical ophthalmic preparations may contain substances that threaten the stability of the delicate two-phase tear film. Benzalkonium chloride (BZA) is commonly used as a preservative in eyedrop preparations and has been regarded by some as the most suitable antimicrobial substance for this purpose (Brown, Foster, Norton, and Richards, 1964). Since BZA is a surface-active agent (and indeed may owe its antibacterial activity at least in part to this detergent property), the present experiments were undertaken to examine its effect on the stability of the tear film in rabbit and man. A significant hastening effect of BZA on corneal drying was observed in both species.

\section{Methods and results}

RABBIT

Dutch rabbits were anaesthetized with halothane ( 3 per cent) in nitrous oxide-oxygen $(3: 1)$. The animals to be tested were placed in front of a small fan to hasten drying of the cornea, and any tendency of the eyelids to droop was overcome by clipping back the fur on to the top of

Address for reprints: Dr W. S. Wilson, Department of Pharmacology, University of Glasgow, Glasgow G12 8QQ the skull. The eyes were equally illuminated by similar 60 watt lamps placed on each side of the animal.

Saline ( 3 drops of 0.9 per cent) was applied to both eyes at zero time; the excess fluid was removed from the conjunctival sac by means of paper tissue without allowing the eyelids to blink. The time taken for the development of a dry spot on each cornea was measured and this was taken as the zero-time control value. The tear film was immediately restored by blinking the eyelids three times. BZA solution ( 3 drops of 0.0001 per cent $w / v$ in 0.9 per cent saline) was then applied, the excess fluid removed as before, and the time for the appearance of a dry spot measured again. This procedure was repeated for successive threefold increases in concentration of BZA. Finally, the cornea was irrigated with saline, and the eyelids repeatedly blinked during a period of about Io minutes, after which another control determination was made 'end control'.

These experiments were so straightforward that they were mainly carried out by one person. Since the possibility of observer bias existed, a second series of experiments was conducted in which an entirely impartial observer judged the times of dry spot appearance; another observer administered the eyedrops and operated the stop-clocks.

The statistical significance of the results was evaluated using the paired Student's $t$ test.

The dry area usually appeared first as a well-defined pit or spot about $\mathrm{I} \mathrm{mm}$ in diameter. If drying had been allowed to continue, other spots would soon have appeared, enlarged, and coalesced until the entire cornea eventually would have appeared dry and dull. Occasionally, the cornea would dry more uniformly, changing gradually from the normal, highly reflective surface to a dull, finely pitted appearance. Control times for the appearance of dry spots were mainly in the range of $1-4$ min, but occasionally exceeded $6 \mathrm{~min}$. While there was considerable variation from animal to animal there was much less between the left and right eyes of each rabbit and even less between zero-time and end controls.

The data mainly derive from the experiments carried out by one operator. About one-third of the observations were, however, derived from experiments involving the independent observer. Since there was no significant difference between the results from both these series of experiments, the data have been combined and are shown in the Figure. Results are expressed as mean percentages of the zero-time control value and I-bars show standard 


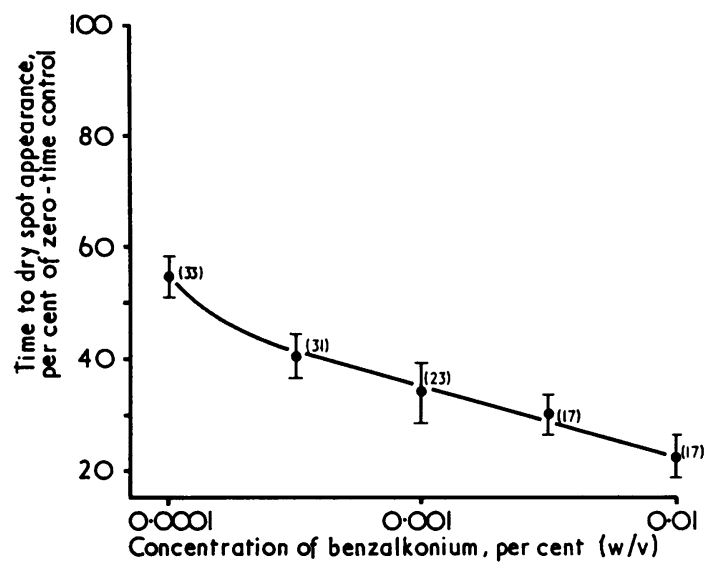

FIGURE Effect of BZA on corneal drying in rabbit. (Data from both series of experiments have been combined)

errors of the means derived from the number of experiments (on individual eyes) shown in brackets.

\section{MAN}

The effect of BZA, at one concentration only, was tested on a group of 16 volunteers, aged between 19 and 40 years (mean $\pm \mathrm{SEM}=25 \cdot 3 \pm \mathrm{r} \cdot 8$ ). Each subject was treated with two drops of oxybuprocaine hydrochloride $(0.4$ per cent $w / v$, Minims Benoxinate) introduced into the conjunctival sac 2-3 $\mathrm{min}$ before the experiment began. Two drops of a sterile solution of either normal saline or BZA (0.01 per cent $w / v$ in saline) were then similarly administered to either the left or right eye (chosen according to a randomly selected protocol card). After blinking a few times and blotting away excess fluid, both eyes were kept open voluntarily and observed for the appearance of focal (F) dry spots or generalized (G) drying of the corneal surface using the slit lamp. The order of application of the two solutions and the order of left and right eye treatment were randomized. In most cases the experiment was terminated after $120 \mathrm{~s}$, whether or not drying had occurred. In some subjects focal drying occurred and observations were continued until the extent of drying became generalized. A second trial was carried out on each subject at least 10 min after the first, both the order of application of the solution and the nature of left and right eye treatment being reversed.
In calculating the difference between the two treatments, comparison was made only between paired times for $F$ degree of drying or between paired times for $G$ drying (that is, an F result was never compared directly with a $G$ result). On this basis, we obtained the results shown in the Table indicating statistically significant hastening of corneal drying by BZA. Similar differences (not shown) are present whether we compare left $v$. right in the first trial combined with left $v$. right in the second trial, or if we compare left in the first with left in the second trial, combined with right in the first compared with right in the second trial. (Both of these methods compared saline with BZA times, as treatments on left and right eyes were reversed in the second trial.) There was a significant $(\mathrm{P}<0.02)$ shortening (by 20.5 s) of the drying time between the first and second trials, irrespective of the nature of the treatment. There was, however, no significant difference between the saline and BZA treatments (applied in the first trial) in their ability to influence the result of the second trial.

\section{Discussion}

BZA shows a remarkable ability to hasten corneal drying in the rabbit, the rate of drying being increased four-fold by a concentration of 0.01 per cent. The effect is dose-related within the range of concentrations tested. The highest of these concentrations, 0.01 per cent is recommended for preservation of many BPC eyedrop formulations, including Hypromellose eyedrops, which are intended to combat corneal dryness. Many contact lens wetting solutions and some proprietary eyedrop formulations include $0.002-0.005$ per cent $\mathrm{BZA}$, but clearly such concentrations are also deleterious to stability of the rabbit's tear film.

The most likely mechanism of this effect is that BZA disrupts the oily layer which normally spreads over the watery lacrimal film. This oily layer derives from the Meibomian glands and has been variously claimed to reduce the evaporation rate from the corneal surface by a factor of about 15 (Mishima and Maurice, 196I) or a factor of four (Iwata and others, 1969). Being a cationic detergent, BZA may well solubilize this extremely thin oily layer, thereby permitting free evaporation of water vapour and hence hastening drying.

Table Effect of BZA on corneal drying in man

\begin{tabular}{|c|c|c|c|c|}
\hline \multirow{2}{*}{$\begin{array}{l}\text { Degree of drying } \\
\text { observed }\end{array}$} & \multirow{2}{*}{$\begin{array}{l}\text { No. of } \\
\text { paired } \\
\text { data }\end{array}$} & \multicolumn{2}{|c|}{ Drying time $(s)($ mean $\pm S E M)$} & \multirow{2}{*}{$\begin{array}{l}P \text { value } \\
\text { of } \\
\text { difference }\end{array}$} \\
\hline & & Saline & $B Z A$ & \\
\hline $\begin{array}{l}F \text { and } G \text { data } \\
\text { combined }\end{array}$ & $3 \mathbf{I}$ & $\begin{array}{l}85 \cdot 4 \pm 8 \cdot 1 \\
(13 \text { eyes }>120 \text { s) }\end{array}$ & $\begin{array}{l}44 \cdot 9 \pm 5 \cdot 3 \\
(2 \text { eyes }>120 \mathrm{~s})\end{array}$ & $<0.001$ \\
\hline G data alone & 24 & $\begin{array}{l}8 \mathrm{r} \cdot \mathrm{I} \pm 8 \cdot 7 \\
(10 \text { eyes }>\text { I } 20 \mathrm{~s})\end{array}$ & $\begin{array}{l}43.5 \pm 6.5 \\
(2 \text { eyes }>120 s)\end{array}$ & $<0.001$ \\
\hline
\end{tabular}


The fact that the rabbit blinks less frequently than the human perhaps indicates that the tear film in man is less effectively protected by an oily layer in the normal condition and would, therefore, be less susceptible to the detergent effect of BZA. Such a deduction is supported by the data presented, since the magnitude of the effect in the rabbit (approximately fourfold hastening of the drying time) was greater than that in man (approximately twofold).

Alternatively or in addition, BZA may encourage dissolution of the conjunctival mucin layer adsorbed on the surface of the corneal epithelium; this would result in an increase in surface tension at the epithelial surface (Lemp and others, 1970), causing the tear film to retract from the affected areas, which then appear as dry spots.

The clinical and pharmaceutical implications of these preliminary results are that BZA does not appear to be a suitable bacteriostat for eyedrop preparations for use in local anaesthesia, and possibly also in keratoconjunctivitis sicca. Lemp, Goldberg, and Roddy (1975) have recently published data on the relative effectiveness of various proprietary artificial tear preparations in prolonging the break-up time of the precorneal tear film. It may be that constituents of these preparations other than their polymer component may be important in their effect on the precorneal film.

This work is currently being extended to investigate the effects of BZA and of a variety of other preservative substances more fully, both in the rabbit and in man.

\section{Summary}

Benzalkonium chloride, a surface-active preservative commonly used in eyedrop preparations, has been shown to hasten the drying of the precorneal tear film. In the rabbit, 0.01 per cent benzalkonium (the concentration usually employed as a preservative) shortened the time required for the appearance of dry spots on the corneal surface by a factor of about four. In man, an approximately twofold hastening was demonstrated. This effect is thought to preclude the use of this substance as a preservative in eyedrop preparations for use as local anaesthetics.

This work was supported in part by Wellcome Trust Grant No. 4896/I.5 to WSW. The authors are grateful to Professor W. S. Foulds for helpful discussions of the problem.

\section{References}

BRoWn, M. R., fOSTER, J. H., NORTON, D. A., and RICHARDS, R. M. (1964) Pharm. $\mathcal{F}$., 192, 8

IWATA, S., LEMP, M. A., HOLlY, F. J., and DOHLMAN, C. H. (1969) Invest. Ophthal., 8, 61 3

LEMP, M. A., GOLDBERG, M., and RODDY, M. R. (1975) Ibid., 14, 255

, holly, F. J., IWATA, S., and Dohlman, c. H. (1970) Arch. Ophthal. (Chicago), 83, 89

, Mishima, s., and maUrice, D. M. (I96I) Exp. Eye Res., 1, 39 